\title{
Estrategia para la evaluación orientada a la formación de la competencia trabajo virtual en equipo en docentes universitarios
}

\author{
Dr. Orosmán Estévez Ariasa, Dra. María de los Angeles Legañoa Ferráb, Dr. Fermín \\ Hurtado Curbelo ${ }^{c}$ \\ a'Universidad de Camagüey Ignacio Agramonte Loynaz, Cuba, orosman.estevez@reduc.edu.cu

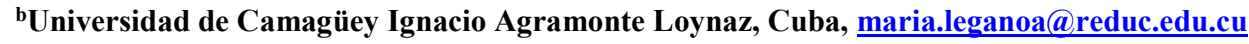 \\ 'Universidad de Camagüey Ignacio Agramonte Loynaz, Cuba, fermin.hurtado@reduc.edu.cu
}

\begin{abstract}
Teamwork is generally defined as a generic competence, built upon from initial stages up to higher education circles. However, some studies have shown that this has not generally been a systematic or regulated subject in the training of college professors, which has resulted in diverse degrees of development. Likewise, the development of Information and Communication Technologies has brought along a set of transformations in the way teamwork isapproached, namely the emergence of virtual teams. Thus, in keeping with the above stated considerations, the paper here presented, a doctoral thesis, aims at promoting and improving the performance of college professors in a teamwork setting. This entails the integration of Web 2.0 tools through an evaluation strategy aimed at the development of the virtual team work competence. The approach was further validated in practice through expert criteria, along with a pedagogical pre-experiment conducted in the Educational Computing School at the Ignacio Agramonte y Loynaz University, which brought along significant advances and transformations in the professors' mode of action .
\end{abstract}

Keywords: teacher training, competence, teamwork, evaluation, strategy

\begin{abstract}
Resumen
El trabajo en equipo es una competencia genérica que se va formando desde niveles precedentes hasta el nivel superior. Sin embargo, estudios realizados revelan que generalmente no ha sido objeto de formación sistémica y regulada en los docentes universitarios, por lo que su grado de desarrollo es muy diverso. A su vez, el desarrollo de las Tecnologias de la Informacion y la Comunicacion (TIC) ha propiciado transformaciones en el trabajo en equipo con el surgimiento de los equipos virtuales. En correspondencia con lo anterior, el trabajo que se presenta, resultado de una tesis doctoral, tiene el objetivo de favorecer el desempeño de los docentes universitarios en el trabajo en equipo. Esto se alcanza con la integración de las herramientas de la Web 2.0 a través de una estrategia de evaluación orientada a la formación de la competencia trabajo virtual en equipo. La estrategia fue validada en la práctica a través del método de criterio de expertos así como en un pre-
\end{abstract}


experimento pedagógico desarrollado en la carrera de Informática Educativa de la Universidad "Ignacio Agramonte y Loynaz",obteniéndose avances y transformaciones significativas en el modo de actuación de los docentes.

Palabras clave: formación de docentes, competencia, trabajo en equipo, evaluación, estrategia.

\section{Introducción}

El Ministerio de Educación Superior (MES) de Cuba, en el marco de la política trazada por el país en torno a la informatización de la sociedad, impulsa la integración de las Tecnologías de la Información y la Comunicación (TIC) a los procesos universitarios con el objetivo de elevar la eficacia y calidad de los mismos. La Dirección de Informatización del MES gestiona la política y recursos para lograr la integración efectiva de las TIC.

Entre las nuevas transformaciones de la Educación Superior Cubana, dirigidas a perfeccionar el sistema de formación continua de los profesionales cubanos, se orienta la aplicación gradual de un nuevo modelo de educación a distancia basado en una pedagogía moderna y en las TIC. En este ámbito, el MES reconoce que es necesaria la integración armónica del quehacer académico y administrativo a fin de lograr, mediante el trabajo en equipo, una formación válida, pertinente y de calidad realizada en espacios y tiempos diferentes, así como la articulación del trabajo colaborativo, en equipos multidisciplinarios, que soportan el proceso de formación continua.

El trabajo colaborativo en la red se ha visto favorecido con la aparición del software social o Web 2.0. Estos desarrollos de web interactivos han posibilitado desarrollar una arquitectura de participación, intercambio y cooperación a través de aplicaciones y servicios abiertos. (Amat, 2011). Con la Web 2.0 la red se ha transformado en una plataforma en la que se crea contenido, se comparte y se reutiliza la información de forma colaborativa.

En la actualidad, para poder realizar los diferentes procesos o llevar a cabo las diferentes tareas en las organizaciones, se precisa de la colaboración y cooperación de varios miembros, estimulando de este modo la participación y la comunicación entre ellos y generando una mejora y un incremento de la calidad. (Park, Henkin, y Egley, 2005). El trabajo en equipo ha ido en aumento desde los años 80 y al presente se reconoce como una competencia genérica a formar en los profesionales.

La competencia trabajo en equipo ha sido identificada por múltiples investigadores (Villa y Poblete, 2010; Bozu y Canto, 2009; Tobón, 2013;Casanellas, 2015; Mas, París y Torrelles, 2016) como una de las competencias genéricas más importantes a desarrollar en la formación continua de los profesionales en sentido general y de los profesionales de la educación en particular. Por su parte Fernández (2011) la identificó como muy necesaria para poder enfrentar los retos de la sociedad del conocimiento y las exigencias del nuevo enfoque curricular basado en competencias, lo cual tiene como fundamento las potencialidades de esta competencia para la formación, desarrollo y evaluación de otras competencias y para activar todo el proceso de actividad comunicación que caracteriza el proceso formativo. 
Diversos investigadores han abordado la estructura de la competencia. Stevens y Campion (1994), identificaron como dimensiones esenciales las de relaciones interpersonales en el equipo y la de autogestión de las tareas para el trabajo en equipo, las cuales han sido asumida por muchos investigadores como Torrelles y otros (2011), Aguado y otros (2014). Los autores del presente trabajo asumen que las mismas deben ser partes componentes de la estructura de la competencia trabajo virtual en equipo.

El desarrollo de las TIC ha provocado transformaciones en el trabajo en equipo originando el surgimiento de los equipos virtuales o virtual teams. El trabajo en equipo se ha visto favorecido con el soporte de las tecnologías colaborativas que promueven la comunicación y el intercambio de información.

Autores como Andres (2012), Devonshire y Henderson (2012) coinciden en afirmar que la asunción del trabajo en equipo con alto nivel de uso de los recursos infotecnológicos, es una alternativa viable y necesaria para desarrollar los procesos de alto grado de autonomía y creatividad.

A partir de los presupuestos anteriores, los autores de la presente investigación definen la competencia trabajo en equipo como una actuación integral de los docentes para colaborar, interactuar e intercambiar información en la realización de actividades interdependientes guiados por un objetivo común en el abordaje de problemas académicos y sociales inherentes al contexto universitario, a través del uso de las TIC, en particular de las herramientas de la Web 2.0, con responsabilidad, compromiso, respeto, constancia y la aplicación de la metacognición. Son dimensiones de esta competencia el establecimiento de relaciones interpersonales entre los miembros del equipo para la comunicación y colaboración, la autogestión para la realización de las tareas en equipo y el manejo de las TIC, en particular de las herramientas de la Web 2.0, para la realización del trabajo en equipo. En estas dimensiones se expresan los criterios de desempeño que la caracterizan.

A partir de estos hallazgos se realizó un estudio preliminar sobre el desempeño de los profesores en ejercicio de la Universidad Ignacio Agramonte Loynaz en el trabajo en equipo, el cual arrojó insuficiencias tales como:

- Insuficiencias en las relaciones interpersonales durante el trabajo en equipo.

- Uso limitado de las TIC para desarrollar los procesos de comunicación durante el trabajo. Se privilegia el correo electrónico y no se utilizan herramientas de la Web 2.0 disponibles para el trabajo colaborativo.

- Se manifiestan dificultades para colaborar y retroalimentar a los miembros de equipos en la realización de las tareas.

- Los directivos hacen uso de las TIC, pero no de las herramientas colaborativas. No intencionan en su gestión el trabajo virtual en equipo.

- No se evidencian acciones formativas dirigidas a integrar las tecnologías de la Web 2.0 al trabajo en equipo.

En general se puede apreciar que la informatización de los procesos que demanda la Educación Superior cubana no se manifiesta en el trabajo en equipo en red en las comunidades académicas. 
Lo anteriormente explicado permitió identificar como problema científico el siguiente: insuficiencias en la formación de docentes universitarios para el trabajo en equipo con herramientas de la Web 2.0 que limitan su desempeño virtual colaborativo.

\section{Objetivo}

En la última década diversos estudios como los de López (2006), Fernández (2011); Hernández, Tobón, y Guzmán (2015) entre muchos, han develado la relación entre la evaluación y la formación de competencias. Han demostrado que la evaluación orientada al aprendizaje suele mejorar considerablemente la motivación e implicación del sujeto en el proceso de formación, contribuyendo a mejorar el mismo, así como los procesos de superación permanente que se desarrollan en la Universidad. Esta evaluación se constituye como el cambio necesario y cualitativo a nivel educativo, que permite modificar la función cuantitativa de la evaluación y la convierte en una herramienta o instrumento importante para el aprendizaje. (Ruíz, 2014)

Por otra parte, se han realizado diversos estudios en el ámbito de la evaluación de la competencia trabajo en equipo, (Torrelles et all, 2015; Torrelles, 2011; Viles, et all, 2012), los cuales ofrecen aportaciones sobre los criterios, métodos e instrumentos a utilizar en la evaluación de la competencia, destacándose en estos el uso de rúbricas, e -rúbricas, portafolios y e-portafolios, así como el empleo de las TIC para el desarrollo de la evaluación. Sin embargo, los estudios realizados no develan su aplicación en la formación continua de docentes, ni toman en cuenta en sus criterios lo referido al desempeño de los docentes en los entornos tecnológicos con el empleo de herramientas de la Web 2.0.

En correspondencia con lo anterior se asumió como objetivo del trabajo que se presenta: favorecer el desempeño de los docentes universitarios en el trabajo en equipo con la integración de las herramientas de la Web 2.0 a través de una estrategia de evaluación orientada a la formación de la competencia trabajo virtual en equipo.

\section{Desarrollo de la innovación}

La formación de la competencia trabajo virtual en equipo (TVE) se concibió fuera realizada a partir de la evaluación de la misma. El proceso debía iniciar con una autoevaluación del docente sobre los desempeños en cada dimensión de la competencia para identificar las necesidades formativas que tenía. La formación se concibió entonces como un proceso de autoformación a partir del metaconocimiento de sus necesidades formativas, el cual era completado durante su desempeño virtual como miembro de un equipo en la solución de problemas académicos o sociales emanados y supervisados desde instancias directivas.

El proceso de formación tendría lugar en un ecosistema de aprendizaje mediado por las TIC, con la participación de especialistas y directivos en un rol formativo, y con el intercambio entre los participantes en los procesos de colaboración y reflexión. Se dispondrían de cursos con ayudas pedagógicas referidas a las dimensiones de la competencia y de evaluaciones continuas (coevaluaciones y heteroevaluaciones) en todo el proceso, para guiar a los docentes hacia un nivel de desarrollo superior de la competencia. La reflexión sobre la práctica y sobre sí mismo que hacen los docentes, son recursos esenciales para la formación de la 
competencia. La evaluación y acreditación de los resultados de la formación en función del nivel de dominio alcanzado y las orientaciones para su mejora son procesos concebidos para el perfeccionamiento continuo del desempeño de los docentes en el trabajo virtual en equipo.

Para instrumentar esta concepción se elaboró una estrategia para la evaluación orientada a la formación de la competencia trabajo virtual en equipo (TVE) en docentes universitarios. Los principales actores de la estrategia fueron un coordinador general para la formación de la competencia TVE, especialistas para la formación de los saberes relativos a las dimensiones de la competencia, directivos designados en las unidades académicas para dirigir el proceso de integración de saberes y los docentes universitarios que están implicados en el proceso de formación de la competencia TVE.

La estrategia fue concebida para que la formación se desarrolle en 2 niveles:

Nivel central: Se desarrollarán acciones formativas por los especialistas soportadas por un sistema de gestión del aprendizaje (LMS) para la formación de conocimientos, habilidades y actitudes (CHA) correspondientes a cada una de las dimensiones de la competencia: establecimiento de relaciones interpersonales entre los miembros del equipo para la comunicación y colaboración, autogestión para la realización de las tareas en equipo y manejo de las TIC, en particular de las herramientas de la Web 2.0, para la realización del trabajo en equipo.

Nivel de unidad académica: se realizarán acciones formativas por los directivos académicos para la integración de saberes a través de la actuación que realizan en la resolución de un problema en equipo, a partir de asumir la virtualidad como elemento esencial del contexto en que desarrollan su proceso formativo y desde donde desarrollan el trabajo en equipo. El problema a resolver presentado por el directivo académico es de significación para los docentes y demanda de la actuación de los mismos a partir del TVE. Esta exigencia de TVE lleva a los docentes involucrados a asumir una tarea para la cual deben poseer una determinada formación de la competencia TVE y cuya identificación se constituye en la base $\mathrm{y}$ acción inicial del proceso formativo.

En la solución del problema en equipo, y también como resultado del proceso de evaluación continua, se produce la identificación de nuevas necesidades de información por el docente para actuar conforme al desempeño deseado, lo que hace que tenga que realizar un proceso de autoformación de los CHA relativos a las dimensiones de la competencia. Se produce entonces una regulación del aprendizaje a partir de establecer nuevas rutas de aprendizaje que den respuestas a estas nuevas necesidades formativas. Este proceso se caracteriza por una interrelación continua entre la formación en el desempeño en el TVE, la autorregulación del aprendizaje y la autoformación de los saberes relativos a las dimensiones que demanda el desempeño.

Para poder implementar esta evaluación orientada a la formación de la competencia TVE es necesario realizar un conjunto de acciones dirigidas a la preparación de los recursos y condiciones para la implementación como son: la selección de las unidades académicas participantes y especialistas para desarrollar la formación, la realización de talleres de 
motivación, la preparación de los directivos que van a dirigir la formación en cada unidad académica, entre otras.

Por ende, en la estrategia se distinguen cuatro fases fundamentales: la fase de preparación, la fase de evaluación diagnóstica, la fase evaluativa-formativa de la competencia y la fase de evaluación, acreditación y orientación las cuales se representan a continuación:

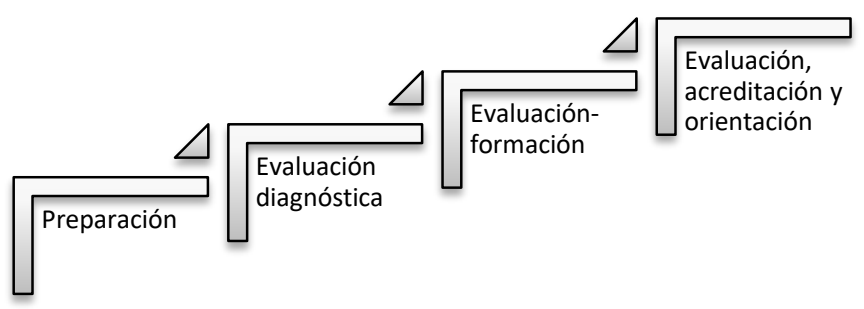

Fig. 1 Fases de la estrategia

Fase de preparación: En esta fase se realizan acciones para la preparación de los recursos y condiciones para la implementación de la estrategia.

Fase de evaluación diagnóstica: En esta fase se realiza la autoevaluación de los profesores participantes en la formación mediante las e-rúbricas. Los resultados posibilitan la proyección de la formación en instancias centrales y en las unidades académicas involucradas. Así como la elaboración de las rutas de aprendizaje por los profesores participantes a partir de las e-rúbricas.

Fase de evaluación-formación: A nivel central se ofrecen los cursos y talleres para la formación en el ámbito del empleo de las TIC, la autogestión y relaciones interpersonales en el TVE. En la unidad académica se desarrolla el trabajo virtual en equipo en un proyecto grupal relativo a un problema del contexto. Los profesores elaboran su e-portafolio con sus aportaciones al proceso, así como las reflexiones en torno al trabajo virtual en equipo. En todo el proceso se desarrolla la evaluación continua de los profesores participantes a través de las e-rúbricas, e-portafolio y otros recursos, que posibilita la retroalimentación continua para la formación.

Fase de evaluación, acreditación y orientación: En esta fase se producen dos procesos, la evaluación y acreditación del nivel alcanzado en la competencia y la orientación de la formación para un nivel superior y/o la mejora contininua.

\section{Valoración de la factibilidad de la estrategia de evaluación orientada a la formación de la competencia trabajo virtual en equipo.}

Para valorar la factibilidad de la estrategia se utilizó el método de criterio de expertos. Los resultados obtenidos mediante el criterio de expertos muestran que: el $86,6 \%$ de los experimentos consideran como muy adecuada la estructura de la competencia trabajo virtual en equipo, mientras que el 14,6\% la considera como bastante adecuada.

Respecto a la orientación de la formación de la competencia trabajo virtual en equipo a partir de la evaluación del nivel de desarrollo de la misma en los docentes universitarios, el 93,3 \% 
de los expertos la consideran como muy adecuada, el 3,3\% la consideran bastante adecuada y el 3,3\% restante la considera adecuada. En este indicador un experto considera que la formación de la competencia a partir de la evaluación de nivel de desarrollo en los docentes universitarios es una vía más entre muchas otras para formar y desarrollar las competencias y expresa sus dudas respecto a la viabilidad de uso de las tecnologías con estos fines en el contexto universitario actual.

En relación a la contribución de la estrategia para favorecer a la formación de la competencia trabajo virtual en equipo en docentes universitarios el $96,3 \%$ la considera como muy adecuada mientras que el 3,3\% restante la valora como bastante adecuada.

A manera de generalización se aprecia que los aspectos sometidos a criterio de los expertos tienen aceptación ya que ninguno de ellos resultó evaluado en las categorías de inadecuado o poco adecuado. El $50 \%$ de los aspectos sometidos a consideración fueron evaluados, por el $100 \%$ de los expertos, en las categorías de muy adecuado y bastante adecuado y el otro 50 $\%$ restante fueron evaluados, por el $96.7 \%$ de los expertos, en las dos categorías anteriores.

Valorada la factibilidad de aplicación de la estrategia para la evaluación orientada a la formación de la competencia trabajo virtual en equipo (TVE), atendiendo al criterio de los expertos consultados, se procedió a la realización de un pre-experimento pedagógico.

\section{Pre-experimento pedagógico para valorar la efectividad de la estrategia}

Para el análisis de los efectos de la aplicación de la estrategia conformada se empleó un enfoque investigativo cuasiexperimental y de carácter longitudinal donde la recopilación de los datos pertinentes se efectuaría por medio de de las erúbricas, de la observación de desempeños de los docentes en el trabajo virtual en equipo efectuada por los directivos que tenía la participaban en la formación y, del análisis del trabajo desarrollado por los docentes contenido en la plataforma del software que se utilizara como sporte al trabajo virtual.

Durante el semestre enero- junio del año 2018 fue llevado a cabo un pre-experimento en la Carrera de Licenciatura en Educación Especialidad Informática, del Departamento de Informática Educativa, perteneciente a la Facultad de Informática y Ciencias Exactas de la Universidad de Camagüey Ignacio Agramonte Loynaz. Se perseguía el objetivo de verificar la factibilidad y efectividad de la estrategia de evaluación para la formación de la competencia TVE durante el desarrollo del trabajo docente metodológico de la Carrera Licenciatura en Educación Especialidad Informática.

En la fase de preparación se hizo un estudio de los recursos informáticos disponibles en la intranet de la universidad a disposición de la comunidad universitaria. Se decidió utilizar el software denominado Redmine como soporte al trabajo virtual en equipo ser un software libre destinado a facilitar el control y seguimiento de proyectos. Redmine ofrece un conjunto de herramientas como son: el calendar, diagramas de gant, las wiki, los foros, los archivos, el visor del repositorio de control de versiones, RSS, control de flujo de trabajo basado en roles, integración con el correo electrónico, administración de noticias, documentos y archivos entre otras opciones, que facilita la planificación de tareas y potencian la comunicación e interacciones que se pueden establecer entre los miembros de un equipo de trabajo virtual. 
Otro de los recursos identificados para la estrategia fue la Nube Reduc, a la cual se puede acceder desde los Servicios REDUC que están en la intranet. Se tuvo en cuenta que la nube posibilita utilizar y compartir diferentes recursos como documentos, rúbricas y portafolios electrónicos que forman parte de los recursos de los que se disponen para facilitar el trabajo colaborativo y el intercambio de información.

En esta fase las acciones estuvieron encaminadas en tres (3) direcciones principales: preparación de los directivos que participarían en la estrategia, elaboración de las rúbricas y e-rúbricas; y elaboración de los cursos a implementar en el entorno virtual.

Para la preparación de los directivos que participarían en la estrategia, el coordinador realizó un taller de motivación con el jefe del departamento y el jefe de la Carrera Licenciatura en Educación Especialidad Informática, con el propósito de motivarlos hacia la formación de la competencia TVE.

El Departamento de Informática Educativa posee una plantilla de 31 profesores, agrupados en cuatro disciplinas: 1) Elementos de Informática, 2) Sistemas de Aplicación, 3) Lenguajes y Técnicas de Programación, 4) Formación Laboral Investigativa. Mediante el método de muestreo no probabilístico en su variante intencionada se seleccionó el colectivo de Carrera, Licenciatura en Educación Especialidad Informática, compuesto por siete profesores los que representan el $22,5 \%$ de la muestra.

Los directivos identificaron como problema a resolver en el equipo la elaboración de la Estrategia de Educación Ambiental para la carrera. Esta estrategia se caracteriza por la especificidad de las acciones a desarrollar en cada disciplina y asignatura. Se sugiere abordarla desde el conocimiento que deben poseer los estudiantes sobre los principales problemas medioambientales que afectan al planeta mediante la búsqueda y procesamiento de la información, la asunción de posiciones reflexivas y críticas sobre los problemas existentes, las causas que los provocan y las medidas que se deben tomar para evitarlos. Esto puede tener diferentes salidas prácticas en dependencia de la disciplina y asignatura en que se trabaje. El trabajo en equipo de los profesores permitirá no repetir acciones y lograr una secuencia lógica en el desarrollo de las mismas, de ahí su importancia.

La segunda dirección de trabajo fue la elaboración de los instrumentos para el diagnóstico y evaluación del progreso de formación de la competencia. En esta etapa se diseñaron y elaboraron las e-rúbrica con el objetivo de diagnosticar el nivel de dominio de la competencia trabajo virtual en equipo que tenían los docentes y que permitiera controlar el progreso de su formación. Para su elaboración se tomaron los criterios de desempeño definidos para esta competencia, correspondientes a las dimensiones de la misma. Se hicieron dos rúbricas, una para la autoevaluación y otra para la coevaluación. La rúbrica de coevaluación posibilitaba que los miembros del equipo evaluaran a sus colegas del equipo, así como permitía ver las evaluaciones que habían hecho los colegas del equipo sobre cada miembro. La elaboración de las rúbricas se hizo en su primera etapa en Excel, para luego elaborar las e-rúbrica correspondientes. Además, se realizó la coordinación con la Dirección de Informatización de la Universidad para crear una categoría denominada "Competencia trabajo virtual en equipo" en el entorno virtual de aprendizaje del postgrado denominado Centro Virtual para el 
Postgrado Universidad de Camagüey (https://moodlepost.reduc.edu.cu). En esta categoría se ubicaron las e- rúbricas y se crearon los cursos definidos.

La tercera dirección en la planificación fue la creación de los cursos. Los especialistas seleccionados como profesores diseñaron los cursos que constituirían el soporte pedagógico para el proceso de formación de la competencia TVE en directivos designados y profesores implicados. Se determinó elaborar tres cursos: (1) que abordara las relaciones interpersonales para el trabajo en equipo; (2) relacionado con la autogestión para la realización de las tareas, para estos dos cursos se designó a una psicóloga como profesora principal; (3) el empleo de las herramientas tecnológicas y de la Web 2.0 para el trabajo en equipo; para este curso se designaron dos especialistas del nodo de la universidad como profesores principales.

Se definió que el diseño de los cursos sobre las tecnologías atendiera a las herramientas disponibles y accesibles por los docentes de la universidad y a los criterios de desempeño. Un módulo se dedicó al uso de las TIC para compartir información y el uso de recursos TIC colaborativos para la creación de documentos (Nube de la universidad, blog, wiki), otro a las herramientas TIC para organizar y planificar el trabajo del equipo, (Calendar), y un tercero dirigido a softwares colaborativos para gestionar proyectos en equipos (Redmine). Este tercer módulo integraba las herramientas anteriores y era la base para el soporte virtual al trabajo por proyectos en equipo. Los especialistas proyectaron el diseño de los mismos a partir de las definiciones hechas.

Esta fase culminó con los talleres que se hicieron con los directivos para prepararlos en relación a los recursos informáticos a utilizar, la competencia TVE y la implementación de la estrategia. Luego el jefe de carrera indicó a los otros seis miembros del colectivo de carrera que debían elaborar la estrategia de educación ambiental de forma colaborativa a través del uso de las TIC.

En la fase de autoevaluación todos los miembros del equipo procedieron a autoevaluar el nivel de desarrollo de la competencia TVE que poseían. Para ello se auxiliaron de la e-rúbrica de autoevaluación disponible en el Centro Virtual para el Postgrado Universidad de Camagüey. De los siete profesores evaluados, dos mostraron un nivel bajo de dominio de la competencia y cinco se ubicaron en el nivel no competente. En la dimensión de relaciones interpersonales seis profesores revelaron estar en el nivel bajo y uno en el medio. En las dimensiones de autogestión para la realización de las tareas y manejo de las TIC, dos mostraron un nivel bajo y cinco mostraron un nivel no competente. Los resultados obtenidos permiten concluir que se evidencian insuficiencias en la autogestión para la realización de las tareas y en el empleo de tecnologías apropiadas para el trabajo virtual colaborativo.

La siguiente fase fue la de evaluación-formación. Una vez identificado el nivel de desarrollo de cada miembro del equipo respecto a la competencia TVE procedieron de manera individual a resolver las dificultades identificadas, para ello hicieron uso del soporte diseñado en el Centro Virtual para el Postgrado Universidad de Camagüey. La autoevaluación inicial de cada individuo se constituyó en el referente de comparación de todo el proceso de evaluación orientada a la formación de la competencia. 
Debido a los mayoritarios resultados de no competencia obtenidos para las dimensiones de autogestión de las tareas y manejo de las TIC, así como para el nivel general de la dominio de la competencia, los esfuerzos se encaminaron a tratar de resolver las dificultades que se habían identificado y para ello se intencionaron acciones que estuvieron dirigidas a dar solución a dichas falencias.

Para atender a las necesidades formativas identificadas en cada una de las dimensiones de la competencia TVE los profesores hicieron uso de los cursos dispuestos en el entorno virtual. En ellos tuvieron acceso a diversos materiales bibliográficos referentes a los contenidos relacionados con los criterios de desempeño. Luego de la lectura de los materiales, pudieron aclarar sus dudas o profundizar en determinados aspectos a través de consultas a los especialistas que atendían los cursos. Las consultas en ocasiones eran personalizadas pero la mayor parte de las veces se produjo un intercambio entre los participantes a través de foros de debate.

Estos foros se convirtieron en un espacio de intercambio de opiniones referente a diversos temas, como fueron la solución de problemas dentro de equipo, cómo mantener una comunicación asertiva, qué acciones realizar para que se produjera la retroalimentación en la realización de las diversas tareas que asume un equipo, la importancia de asumir los objetivos del equipo como propios, lo conveniente de apoyar a los compañeros del equipo en la realización de las tareas, y la necesidad de controlar los resultados del trabajo de cada miembro.

El rol desempeñado durante esta etapa por los especialistas fue de vital importancia, pues garantizó el acompañamiento de los participantes en el curso, y sus intervenciones para aclarar dudas o guiar los debates fue de gran valía. Por otra parte, el coordinador controló la marcha del proceso formativo y garantizó que las condiciones creadas fueran funcionales.

Muy importante en esta fase fue el curso sobre el empleo de las herramientas tecnológicas y de la Web 2.0 para el trabajo en equipo. Este curso permitió capacitar a los docentes en el uso de las mismas y sirvió de espacio para guiar el trabajo de estos en el proyecto en que de modo paralelo participaban. A través de las posibilidades de interacción que se brindaban desde el curso los docentes pudieron consultar de manera permanente a los especialistas que atendían los mismos y ganar en conocimientos y habilidades en el manejo de las diferentes herramientas tecnológicas objeto de estudio.

De forma paralela al proceso de autoformación que emprendieron los docentes se comenzó a trabajar en el desarrollo del Proyecto de Educación Ambiental para la carrera. Todas las tareas acometidas en la realización del proyecto fueron realizadas desde el software Redmine. Este software sirvió de plataforma para la comunicación, evaluación del trabajo de los miembros del equipo, la retroalimentación, consultas de documentos y socialización de los resultados logrados en cada etapa del mismo. Desde el mismo, la líder del proyecto y los seguidores de tareas le dieron seguimiento al cronograma de ejecución.

El jefe del proyecto fue el encargado de asignar las tareas a los diferentes miembros del equipo y además asignaba seguidores, los que daban seguimiento al cumplimiento de las mismas, y al finalizar el período de ejecución de cada una emitían un criterio evaluativo sobre 
el desempeño de los compañeros que las habían ejecutado. Los criterios que emitían los miembros del equipo que se les asignaba el rol de seguidores de tareas estaban orientados a valorar cómo cada sujeto encargado de cumplir una tarea individual o colectiva se desempeñaba en cada una de las dimensiones de la competencia, para ello se guiaban por los criterios de desempeño declarados para cada dimensión y que aparecían en la e-rúbrica de coevaluación. Esta acción estuvo encaminada a resolver uno de los principales problemas que habían sido identificados inicialmente y que está relacionado con la autogestión para la realización de las tareas.

El trabajo en el proyecto se organizó por tareas, unas se realizaron de forma colectiva y otras individuales. En la fase inicial del proyecto todos los participantes acometieron la tarea de estudiar los documentos normativos con el objetivo de realizar el análisis de las potencialidades de los programas de las asignaturas para determinar en qué componente del proceso (académico, laboral, investigativo y extensionista) se debían diseñar acciones para dar salida a la temática medioambiental. Luego separados por disciplinas y asignaturas se realizó el diseño de las acciones que conformarían la estrategia para cada uno de los componentes del proceso de formación. A continuación las acciones diseñadas fueron valoradas por el Colectivo de Carrera y alcanzado el consenso sobre las mismas se procedió a la integración de estas y a la elaboración del documento final. Logrado lo anterior se procedió a la socialización de la estrategia elaborada.

Se orientó la comunicación entre los sujetos a través del Redmine y otros recursos informáticos para realizar la retroalimentación. También se realizó la elaboración colectiva de documentos a través de diferentes herramientas para el trabajo colaborativo.

La e-rúbrica de coevaluación fue muy útil para el proceso de retroalimentación y aportó elementos importantes para el proceso de heteroevaluación, los que se ejecutaron de forma sistemática. Estos procesos aportaron información para cada sujeto sobre los criterios que sobre su trabajo tenían el resto de los miembros del equipo, constituyéndose en la base del perfeccionamiento continuo.

Cada miembro del equipo confeccionó su e-portafolio, para esto se utilizó la herramienta Archivo de Redmine. Con el auxilio de esta herramienta elaboraron sus reflexiones sobre el trabajo desarrollado y valoraron sus aciertos y desaciertos en la ejecución de las diversas tareas colaborativas a través del uso de las tecnologías. Esta acción fue diseñada a partir de considerar que era necesario que los profesores se implicaran en la búsqueda de soluciones a los problemas que enfrentaba el equipo y que de manera mayoritaria había sido identificado como deficiente en el diagnóstico inicial de la dimensión autogestión para la realización de las tareas.

Las reflexiones recogidas en los e-portafolios estuvieron dirigidas a la apreciación que tenía cada sujeto sobre cómo transcurría la formación de la competencia a partir de las valoraciones que realizaban de la formación de cada una de las dimensiones que componen la misma. El análisis de los e-portafolios elaborados permitió a los encargados de la formación de la competencia valorar la apreciación que tenían los sujetos sobre su propio proceso de formación de la competencia TVE en general, y de cada una de las dimensiones de la misma. 
Constituyó un documento importante para identificar además las necesidades formativas no satisfechas.

Las actividades colaborativas se evaluaron a partir de la utilización de la herramienta Wiki del Redmine y del uso de documentos en el repositorio, en el cual podían colocar las producciones propias. La comunicación y colaboración se evaluó a partir de la participación en los diferentes foros que se desarrollaron desde el Redmine. También se tuvieron en cuenta las coevaluaciones realizadas por los miembros del equipo.

Los criterios de los agentes formativos que intervinieron en el proceso de formación de los saberes de la competencia se formaron a partir del análisis del trabajo desarrollado. En el mismo se pudieron apreciar el uso de las diferentes herramientas tecnológicas en el establecimiento de relaciones interpersonales y la autogestión para el desarrollo de las tareas, así como del análisis de los informes de los productos del trabajo realizado en equipo en la resolución de problemas del contexto.

En la fase de evaluación, acreditación y orientación tanto para las dimensiones de la competencia como para el nivel general de la misma, se realizó el análisis de las evidencias de desempeño de cada sujeto declaradas en la competencia, los resultados de la-rúbrica de coevaluación y la heteroevaluación que hizo el directivo al frente del proceso a partir del seguimiento de las tareas. También se consideró la apreciación que tenía el sujeto evaluado respecto a la formación de los diferentes criterios de desempeño de cada una de las dimensiones de la competencia y que se manifestaron en las rúbricas de autoevaluación inicial y final. Se consideró el desempeño de cada integrante en las tareas individuales y colectivas. El criterio evaluativo final que emitió el directivo fue analizado en el equipo de trabajo donde se valoraron los avances logrados y se identificaron aquellos aspectos que eran susceptibles de continuar mejorándose, de esta forma se identificó el punto de partida de un posible nuevo ciclo de formación de la competencia TVE.

En este proceso los encargados de la formación de la competencia y la jefa del colectivo de la carrera jugaron un papel fundamental, pues no solo intervinieron a lo largo de todo el proceso para ajustar los elementos que fueron necesarios, sino que orientaron de forma personalizada los aspectos que se mejoraron y los que debían ser mejorados para alcanzar un dominio alto de la competencia.

De los siete profesores evaluados, seis mostraron un nivel bajo de dominio de la competencia y uno mostró un nivel medio. En la dimensión de relaciones interpersonales seis profesores revelaron estar en el nivel medio y uno en el nivel alto. En las dimensiones de autogestión para la realización de las tareas y manejo de las TIC, cinco mostraron un nivel bajo y dos mostraron un nivel medio.

\section{Resultados}

En la dimensión de relaciones interpersonales se revela que en el 100\% de los profesores se manifiestan avances en los criterios de desempeño de la dimensión, puestos de manifiestos en su actividad durante la realización del proyecto y corroborados por los diferentes momentos de evaluación a que fueron sometidos. 
En la dimensión autogestión para la realización de las tareas se revela que el 71,4 \% de los profesores manifiestan avances en los criterios de desempeño de la dimensión. El resto (28,6 $\%$, no lograron manifestar en el período evaluado avances notables en los criterios de desempeño de la dimensión, esto pudiera ser muestra de la complejidad del proceso de formación de la competencia, de la heterogeneidad de los miembros del equipo y de sus diferencias individuales.

En la dimensión manejo de las TIC, en particular de las herramientas de la Web 2.0, para la realización del trabajo en equipo se revela que el $85,7 \%$ de los profesores logran alcanzar avances notables en los criterios de desempeño de la dimensión. Se destaca que el 14,3\% restante (1 profesor), no logra obtener avances significativos en los criterios de desempeño de la dimensión, es significativo que es el mismo sujeto el que manifiesta las dificultades mayores en dos de las tres dimensiones analizadas. La Prueba de la Suma de Rangos de WILCOXON demostró que las diferencias eran significativas.

La evaluación del nivel general de la competencia reveló que el 71,4\% de los profesores lograron transitar del nivel no competente al nivel bajo de dominio de la competencia, que un profesor (14,2\%) logró transitar del nivel de dominio bajo al nivel de dominio medio y que un profesor $(14,2 \%)$ no logró en el período de tiempo analizado avanzar respecto al nivel de dominio de la competencia. La Prueba de la Suma de Rangos de WILCOXON demostró que las diferencias eran significativas.

Se considera que la competencia trabajo virtual en equipo no solo resulta desarrollada en los docentes como consecuencia de la aplicación de la estrategia, sino también en los directivos, en los especialistas que impartieron los cursos, los que elaboraron las herramientas y los investigadores, quienes se encargaron de manera responsable de asumir y cumplir funciones imprescindibles para el trabajo virtual en equipo.

\section{Conclusiones}

La concepción teórica de la estrategia de evaluación orientada a la formación de la competencia TVE indica las bases de la relación entre la evaluación y la formación. Su coherencia lógica se hace sustentable por la veracidad de las presunciones que conectan sus fases: desde la evaluación diagnóstica se orienta la proyección de la autoformación, la evaluación continua controla el proceso de autoformación y formación colaborativa y la evaluación acreditativa dirigida al mejoramiento continuo.

La estrategia para la evaluación orientada a la formación de la competencia TVE constituye una herramienta que posibilita la instrumentación de las acciones de los actores dirigidas a trascender las limitaciones que exhiben los docentes universitarios en el trabajo virtual colaborativo, a partir del perfeccionamiento de las relaciones interpersonales, la autogestión de tareas y su integración a las herramientas de las Web 2.0 que viabilizan la comunicación e información en el trabajo en equipo, lo cual fue reconocido por los expertos cuando fueros encuestados y validada en la práctica con transformaciones muy significativas en el modo de actuación de los docentes y directivos. 
Estrategia para la evaluación orientada a la formación de la competencia trabajo virtual en equipo en docentes universitarios

\section{Referencias}

AMAT, A. F. (2011). "Usando la web 2.0 para informarse e informar. Una experiencia en educación superior".Teoría de la Educación: Educación y cultura en la Sociedad de la Información, 12, 1, 145-166. <http://revistas.usal.es/index.php/eks/article/view/7827> [Consulta: 8 de noviembre de 2015]

ANDRES, H. (2012). "Technology-mediated collaboration, shared mental model and task performance". Journal of Organizational and End User Computing, 24, 1, 64-81.< http://www.irma-international.org/viewtitle/61413/>[Consulta: 8 de noviembre de 2015]

Aguado, D., et all (2014). "Teamwork Competency Test (TWCT): a Step Forward on Measuring Teamwork Competencies". Group Dynamics: Theory, Research, and Practice, 18, 2, 101-121.< http://dx.doi.org/10.1037/a0036098>[Consulta: 8 de noviembre de 2015]

BOzU, Z., y CANTO, P. J. (2009). "El profesorado universitario en la sociedad del conocimiento:competencias profesionales docentes". Revista de Formación e Innovación Educativa Universitaria, 2,2, 221-231. < http://refiedu.webs.uvigo.es/Refiedu/Vol2_2/REFIEDU_2_2_4.pdf $>$ [Consulta: 8 de noviembre de 2015]

CASANELlas, S. (2015). La competencia transversal de trabajo en equipo. Instrumentos para su implementación y evaluación. Barcelona: Universidad de Barcelona.< https://www.uoc.edu/symposia/dret_tic2012/pdf/3.4.casanellas-montserrat-y-solemarina.pdf $>$ [Consulta: 15 de septiembre de 2017]

DEVONSHIRE, E. R., y HENDERSON, E. (2012). "Reducing the distance: providing challenging and engaging online postgraduate education in pain management". British Journal of Pain, 6, 2, $70-78 .<$ doi: 10.1177/2049463712448582>[Consulta: 8 de noviembre de 2015]

FERNÁNDEZ, A. (2011). "La evaluación orientada al aprendizaje en un modelo de formación por competencias en la educación universitaria". REDU. Revista de Docencia Universitaria, 8, $\quad 11, \quad 11-34 .<$ http://red-u.net/redu/files/journals/1/articles/144/public/144-130-2PB.pdf $>$ [Consulta: 8 de noviembre de 2015]

Hernández, J. S., Tobón, S., y GuZMÁn, C. (2015). "Evaluación Socioformativa y Rendimiento Académico en un Programa de Posgrado en Línea". Paradigma, XXXVI, 1, 30 $-41 .<\mathrm{http}: / /$ revistas.upel.edu.ve/index.php/paradigma/article/view/2652/0>[Consulta: $22 \mathrm{de}$ diciembre de 2016]

LÓPEZ, V. (2006). "El papel de la evaluación formativa en el proceso de convergencia hacia el E.E.E.S.Análisis del estado de la cuestión y presentación de un sistema de intervención”. Revista Interuniversitaria de Formación del Profesorado, 20, 3, 93-105. $<$ http://www.redalyc.org/articulo.oa?id=27411311005 $>$ [Consulta: 8 de noviembre de 2015]

MAS O., PARÍS G., TORRelles C. (2016). "El trabajo en equipo: dominio competencial en diferentes grados de la Universitat Autònoma de Barcelona y de la Universitat de Lleida". Revista de Ciències de l'Educació, No 1, 55-66, https://doi.org/10.17345/ute.2016.1.978 > [Consulta: 15 de septiembre de 2017]

(c)) BY-NC-ND 2019, Universitat Politècnica de València 
PARK, S., Henkin, A. B., y EgLEY, R. (2005). "Teacher team commitment, teamwork and trust: Exploring associations", Journal of Educational Administration. 43,5,115-125. $<$ https://doi.org/10.1108/09578230510615233>[Consulta: 14 de noviembre de 2015]

Ruíz, Y. (2014). Estudio de las competencias transversales en un modelo de enseñanza y evaluación formativa en la universidad. Málaga: Publicaciones y Divulgación Científica. Universidad de Málaga.< http://hdl.handle.net/10630/8171> [Consulta: 24 de septiembre de 2015]

Stevens, M. J., y CAMPION, M. A. (1994). "The knowledge, skill, and ability requirements for teamwork:Implications for human resource management". Journal of Management, 20, 503-530.< https://doi.org/10.1016/0149-2063(94)90025-6> [Consulta: 24 de septiembre de 2015]

TоBÓN, S. (2013). Formación integral y competencias: pensamiento complejo, currículo, didáctica y evaluación. Bogotá: Ecoe.

$<$ https://www.researchgate.net/profile/Sergio_Tobon4/publication/319310793_Formacion_i ntegral_y_competencias_Pensamiento_complejo_curriculo_didactica_y_evaluacion/links $/ 5$ 9a2edd9a6fdcc1a315f565d/Formacion-integral-y-competencias-Pensamiento-complejocurriculo-didactica-y-evaluacion.pdf $>$ [Consulta: 23 de noviembre de 2015]

Torrelles, C. (2011). Eina d'evaluació de la Competéncia de Treball en Equip. Tesis doctoral, Universidad de Lleida. España. < http://hdl.handle.net/10803/51341> [Consulta: 23 de noviembre de 2015]

TORRElles, C., et all. (2011). "Competencia de trabajo en equipo: Definición y Categorización”. Profesorado. Revista de currículum y formación del profesorado, 15, 3, 329-344.<https://www.ugr.es/ recfpro/rev153COL8.pdf> [Consulta: 23 de noviembre de 2015]

VILES, E. et all (2012). "Evaluación de la competencia de trabajo en equipo en los grados de ingeniería". Dirección y Organización, 46, abril, 67-75. $<$ http://www.revistadyo.com/index.php/dyo/article/download/392/412>, [Consulta: 23 de noviembre de 2015]

Villa, A., y Poblete, M. (2011). "Evaluación de competencias genéricas:principios, oportunidades y limitaciones". Revista Bordón, 63,1, 147170. $<$ https://recyt.fecyt.es/index.php/BORDON/article/view/28910> [Consulta: 14 de noviembre de 2015] 